\title{
Notch Signaling Downregulates MUC5AC Expression in Airway Epithelial Cells through Hes1-Dependent Mechanisms
}

\author{
Hai-Feng Ou-Yang Chan-Gui Wu Shuo-Yao Qu Zhi-Kui Li \\ Department of Respiratory Medicine, Xijing Hospital, Fourth Military Medical University, Xi'an, China
}

\author{
Key Words \\ MUC5AC · Notch · Airway mucus hypersecretion . \\ Transcription
}

\begin{abstract}
Background: Mucus overproduction is one of the major pathological features of asthma, and MUC5AC is the major mucin component of airway mucus. However, whether Notch signaling is implicated in the regulation of MUC5AC expression in airway secretary cells is still undetermined. $\mathbf{O b}$ jective: The aim of this study is to examine whether Notch signaling can regulate MUC5AC expression and explore the molecular mechanisms. Methods: Mouse mtCC1-2 cells and human $\mathrm{NCl}-\mathrm{H} 292$ cells were transfected with NIC, and MUC5AC expression was examined. Using gene reporter assays, site-directed mutagenesis, and ChIP assays, the activity of both mouse and human MUC5AC promoter was analyzed. Results: Notch signaling regulated MUC5AC expression both in mouse mtCC1-2 cells and in human NCl-H292 cells. Several Hes-binding site $\mathrm{N}$-boxes were identified in the $5^{\prime}$ region of both mouse and human MUC5AC promoters. Overexpression of NIC resulted in activation of the MUC5AC promoter. Site-directed mutagenesis report assays revealed that Hes proteins might repress both mouse and human MUC5AC promoter activity. Furthermore, ChIP assays confirmed that Hes 1 binds to the MUC5AC promoter both in mouse mtCC12 cells and in human NCl-H292 cells. Conclusions: Notch sig-
\end{abstract}

naling can directly downregulate $M U C 5 A C$ promoter activity through Hes1-dependent mechanisms, which may be identified as possible targets for pharmacotherapy of airway mucus hypersecretion in asthma.

Copyright @ 2013 S. Karger AG, Basel

\section{Introduction}

Mucus overproduction is one of the major pathological features of asthma, which is generally thought to be responsible for small-airway obstruction and lung dysfunction that is closely linked to morbidity and mortality in asthma [1]. However, there is no specific therapy for mucus overproduction in asthma patients at present [2]. The major mucin components of airway mucus are MUC5AC and MUC5B, which contribute to the viscoelastic properties of the mucus [2]. In addition, MUC5AC has been implicated as a marker of goblet cell metaplasia in lung pathologies [3]. Though several studies have linked IL-13 and EGFR pathways to MUC5AC expression [1], more signaling pathways leading to MUC5AC expression remain to be established.

Notch signaling is a highly conserved pathway. Interaction between Notch ligands and receptors triggers a $\gamma$-secretase-mediated proteolysis of the receptors and liberation of the Notch intracellular domain (NIC) into cytoplasm. NIC then translocates into the nucleus, where it binds to and transactivates the transcription factor RBP-J.

\section{KARGER}

E-Mail karger@karger.com

www.karger.com/res
(C) 2013 S. Karger AG, Basel

0025-7931/13/0864-0341\$38.00/0
Hai-Feng Ou-Yang or Zhi-Kui Li

Department of Respiratory Medicine, Xijing Hospital

Fourth Military Medical University

Xi'an 710032 (China)

E-Mail ouyhf@hotmail.com 
This leads to activation of the Notch downstream target genes Hes and Hey. Hes proteins are suppressive basic helix-loop-helix (bHLH) molecules. In the lung, the best characterized Notch target is Hes1 [4].

Several studies have implicated Notch signaling in MUC5AC expression and goblet cell fate, both in development and in disease. Inhibition of Notch signaling by $\gamma$-secretase inhibitors results in an increased number of intestinal goblet cells and MUC5AC expression [5, 6]. Conversely, constitutive Notch activation impairs goblet cell differentiation and MUC5AC expression in the intestine $[7,8]$. In addition, during postnatal life Notch is required to prevent Clara cells from differentiating into goblet cells [9]. However, more studies are needed to determine the exact molecular mechanisms of how Notch signaling regulates MUC5AC expression in airway epithelial cells.

In the present study, we show that Notch signaling directly targets the MUC5AC promoter. Hes1 can bind to the $\mathrm{N}$-boxes (Hes-binding site) located in the $5^{\prime}$ region of the MUC5AC gene. Hes1 is a transrepressor of the MUC5AC promoter in vitro. Furthermore, Hes 1 binds to the MUC5AC promoter both in mouse mtCC1-2 cells and in human NCI-H292 cells. Taken together, our results suggest that MUC5AC expression may be directly regulated by Notch signaling in airway epithelial cells.

\section{Materials and Methods}

\section{Cloning and Construction}

The mouse $M u c 5 a c$ promoter (from $-1,450$ to +132 ) was amplified by PCR using primers $5^{\prime}$-ACTCACTCATTCACAAGT CACTCCTCTA and $5^{\prime}$-ACTTTGAGTCATTACCTGTGCATT GGCTG. The human MUC5AC promoter (from -1,366 to +47) was amplified by PCR using primers $5^{\prime}$-GGCACCTGGGCTA AGACAGGACA and 5' -TGTGTGGACGGCGGGGAAGAGTG. The amplified promoter fragments were cloned into the pGL3basic vector (Promega Life Science, Madison, Wisc., USA) to generate pGL3-mMuc5ac and pGL3-hMUC5AC. Site-directed mutagenesis of N-boxes in the MUC5AC promoter was performed using a QuickChange kit (Stratagene) according to the manufacturer's instructions. Reporter constructs derived from these mutant versions of the MUC5AC promoter were named pGL3-mMuc5acNbox1, pGL3-mMuc5ac-Nbox2, and pGL3-hMUC5AC-Nbox (fig. 3b, c, 4b).

\section{Reporter Assay}

mtCC1-2 cells (mouse transformed Clara cell line) $\left(2 \times 10^{4}\right)$ were cultured in Dulbecco's modified Eagle's medium (DMEM) supplemented with $10 \%$ fetal calf serum (FCS) and $2 \mathrm{mM}$ glutamine (Invitrogen). NCI-H292 cells (human mucoepidermoid cell line) were cultured in RPMI 1640 supplemented with 10\% FCS, 4 mM glutamine, $1 \mathrm{~mm}$ sodium pyruvate, $10 \mathrm{~mm}$ Hepes, and antibiotics. Cells were transfected with $0.1 \mu \mathrm{g}$ reporter construct, $0.1 \mu \mathrm{g}$ pEF-
Bos-NIC-neo $[10,11]$, and 5 ng renilla luciferase vector (phRL-TK; Promega, Madison, Wisc., USA) using Lipofectamine $2000^{\mathrm{TM}}$ (Invitrogen). The total amount of transfected DNA was balanced with pEF-Bos-neo. Forty-eight hours after transfection, luciferase activity was assessed using a Luminoskan Ascent (Labsystems, Helsinki, Finland) and a Dual-Luciferase Reporter Assay kit (Promega) according to the manufacturer's protocol. All luciferase activity was normalized with renilla luciferase activity. Data were analyzed using t tests. $\mathrm{p}<0.01$ and $\mathrm{p}<0.05$ were considered statistically significant.

\section{$R T-P C R$}

Two micrograms of total RNA were reverse-transcribed with reverse transcriptase (Promega) according to the manufacturer's instructions. All PCR experiments were performed with Taq polymerase (Promega) and the primers $5^{\prime}$-ACTGTTACTATGCGA TGTGTAGCCA and $5^{\prime}$-GAGGAAACACATTGCACCGA for mouse MUC5AC, 5' -AGCCCGGCGCCGTGGTCTCCT and 5'GGCGGGGCTCTT GCTGGTGTTGGT for human MUC5AC, 5'-AACCCTAAGGCCAACCGTGAAAAG and 5'-GCAGGATG GCGTGAGGGAGAG for $\beta$-actin control.

\section{Western Blotting}

Proteins were separated by SDS-polyacrylamide gel electrophoresis (SDS-PAGE) and transferred to nitrocellulose membranes. The membranes were saturated for $1 \mathrm{~h}$ at room temperature in TBST supplemented with $5 \%$ skim milk and immunoblotted overnight at $4^{\circ} \mathrm{C}$ with anti-mouse Muc5ac (sc-71620; Santa Cruz Biotechnology, Santa Cruz, Calif., USA), anti-human MUC5AC (sc20118; Santa Cruz Biotechnology), anti-Notch1 (NIC, M-20; Santa Cruz Biotechnology), anti-Hes1 (H-140; Santa Cruz Biotechnology), or anti- $\beta$-actin antibody (I-19; Santa Cruz Biotechnology). Membranes were then washed, probed with horseradish peroxidase (HRP)-conjugated goat anti-rat, rabbit anti-goat, or goat antirabbit polyclonal antibodies (Zhong-Shan Biotech, Beijing, China) according to the first antibody, and revealed with enhanced chemiluminescence (Amersham Biosciences, Uppsala, Sweden).

\section{Chromatin Immunoprecipitation}

Chromatin immunoprecipitation (ChIP) was performed using a ChIP assay kit following the recommendations of the supplier (Upstate Biotechnology). Anti-Hes1 antibodies (sc-13844; Santa Cruz Biotechnology) were used for immunoprecipitation of chromatin along with controls. PCR primers for ChIP assays included: mouse N-boxes, $5^{\prime}$-CTGTCTGTCTCTCTGTCTCT and $5^{\prime}$-CAT TTGAACTTTCTGGTTGC, and human N-boxes, $5^{\prime}$-CATGGG AGGAATGGCAGGA and 5'-TGCCGGGTAGAGCCCAGTT.

\section{Results}

\section{Ectopic Overexpression of NIC Can Regulate}

MUC5AC Expression

To investigate whether Notch signaling can regulate MUC5AC expression, we upregulated Notch signaling in mouse mtCC1-2 cells and detected the expression of Muc5ac. The results showed that the expression of Muc5ac significantly decreased in pEF-Bos-NIC-neo-transfected 


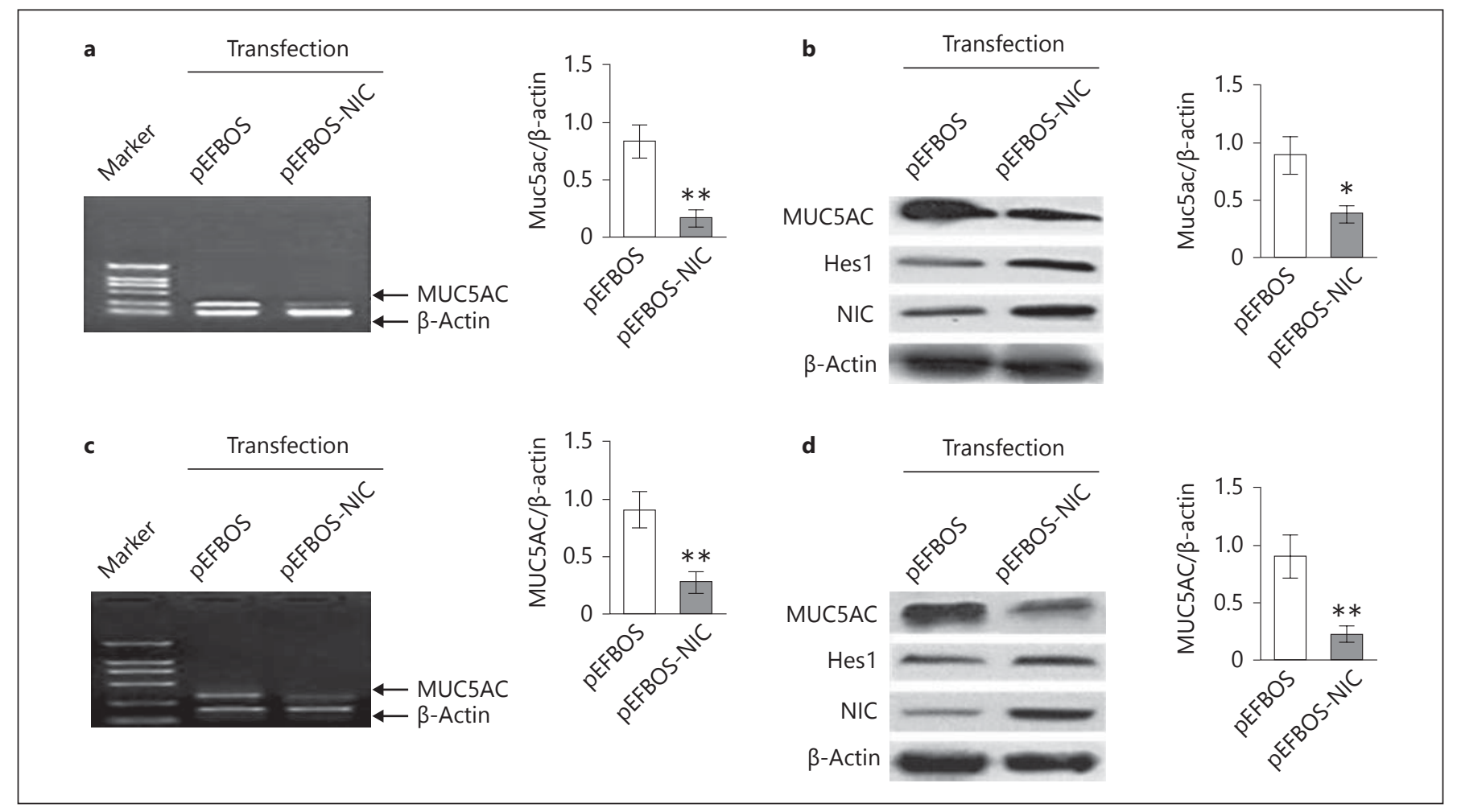

Fig. 1. Ectopic overexpression of NIC regulated MUC5AC expression. a MUC5AC mRNA in the transfected mtCC1-2 cells was detected by RT-PCR with $\beta$-actin as a control $(n=5)$. b MUC5AC, NIC, and Hes 1 proteins in the transfected mtCC1-2 cells were detected by Western blotting with $\beta$-actin as a control for protein

cells both in mRNA level and in protein level compared to pEF-Bos-neo-transfected cells (fig. 1a, b; MUC5AC mRNA, $\mathrm{p}<0.01$; MUC5AC, $\mathrm{p}<0.05)$. Similarly, upregulation of Notch signaling decreased MUC5AC expression in human NCI-H292 cells (fig. 1c, d; MUC5AC mRNA, $\mathrm{p}<0.01$; MUC5AC, $\mathrm{p}<0.01$ ). In addition, the regulatory effects of NIC are dose dependent (data not shown).

\section{Sequence Alignment Analysis of the Human and Mouse MUC5AC Promoters}

To determine whether Notch signaling directly regulated the MUC5AC promoter, we analyzed the genomic sequence of the mouse (NT_039437.8) and human (NT_009237.18) MUC5AC promoters. An alignment of the mouse $M u c 5 a c$ promoter $(-1,864$ to +316$)$ with the human counterpart is depicted in figure $2 \mathrm{a}$, showing the consensus transcriptional start site (TSS) and TATA box of the MUC5AC promoters. In the $5^{\prime}$ region of both mouse and human MUC5AC promoters, we found several Hes consensus binding motifs, defined as N-box (CACNAG) (fig. 2b). loading $(\mathrm{n}=5)$. c MUC5AC mRNA in the transfected NCI-H292 cells was detected by RT-PCR with $\beta$-actin as a control $(n=5)$. d MUC5AC, NIC, and Hes1 proteins in the transfected NCI-H292 cells were detected by Western blotting with $\beta$-actin as a control for protein loading $(n=5)$.

\section{Effects of the Hes-Binding Sites on the Mouse Muc5ac}

\section{Promoter}

To observe whether these putative transcription factor-binding sites modulated transcriptional activity, we amplified the mouse $M u c 5 a c$ promoter $(-1,450 /+312)$ and constructed a reporter plasmid pGL3-mMuc5ac. Cotransfection of mtCC1-2 cells with the reporter and increasing doses of NIC expression vector pEF-Bos-NIC gradually decreased Muc5ac promoter activity (fig. 3a).

To look at the function of the N-boxes in the Muc5ac promoter, we disrupted these N-boxes by site-directed mutagenesis and examined the consequence using the reporter assay (fig. 3b). As shown in figure 3c, mutation of the N-box at position -893 resulted in an increase in promoter activity. However, disruption of the N-box located at $-1,439$ had no influence on the activity of the promoter. These results suggested that Hes proteins might repress mouse $M u c 5 a c$ promoter activity.

Because Hes1 plays a critical role in regulating airway epithelial development, we next investigated whether 
Fig. 2. a Sequence alignment of the human and mouse MUC5AC promoters. TATA box, TSS, and N-boxes are indicated. b Scheme of the $5^{\prime}$ region of the mouse and human MUC5AC promoter, indicating the potential Hes recognition sites.

Fig. 3. Effects of the Hes-binding sites on the mouse $M U C 5 A C$ promoter. a Reporter assay. mtCC1-2 cells were transfected with pGL3-MUC5AC (100 ng) and gradient doses of NIC $(0,60,80$, and $100 \mathrm{ng})$ as well as other plasmids as indicated. The relative luciferase activity (firefly luciferase/renilla luciferase) was analyzed $48 \mathrm{~h}$ later. The results are presented as means \pm SD. ${ }^{* *} \mathrm{p}<$ $0.01(\mathrm{n}=3)$. $\mathbf{b}$ Scheme of the site-directed mutant of the mouse MUC5AC in which the N-boxes were disrupted. c Reporter assay. mtCC1-2 cells were transfected independently with the wild type and deletion mutant of the MUC5AC reporter constructs and were assayed as in figure $3 \mathrm{a}$. d ChIP. Chromatin preparations from mtCC1-2 cells were immunoprecipitated using antiHes1, and co-precipitated DNA fragments were amplified by primers specific for the N-box fragment of the MUC5AC promoter, respectively. Normal goat serum was used as a control. Luc $=$ Luciferase.

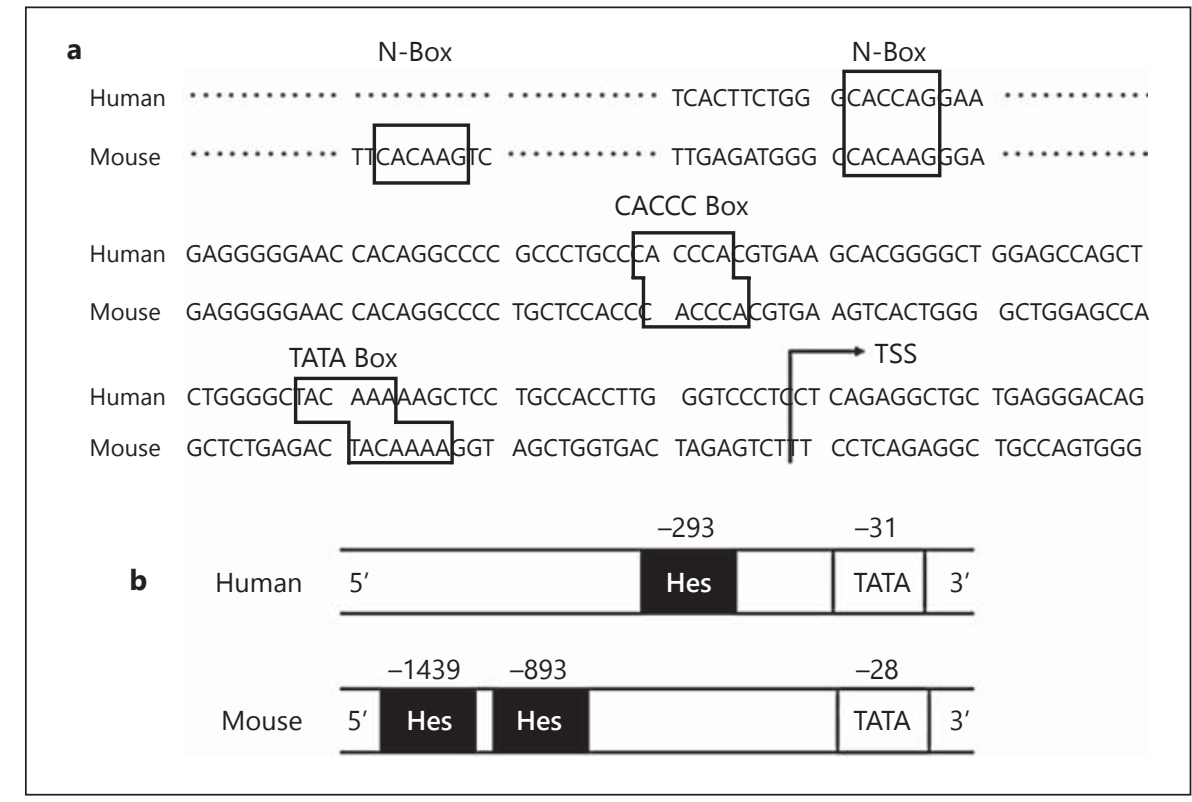

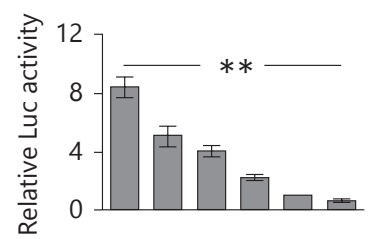

pGL3-mMUC5AC + + + + + +

$\mathrm{phRL}++++++$

pEF-Bos-NIC -

Hes 1

NIC b $\quad-1439 \quad-893$

pGL3-mMuc5ac-Nbox1

pGL3-mMuc5ac-Nbox2

pGL3-mMuc5ac

\begin{tabular}{|c|c|}
\hline Hos & Hes \\
\hline Hes & Hes \\
\hline Hes & Hes \\
\hline
\end{tabular}

c
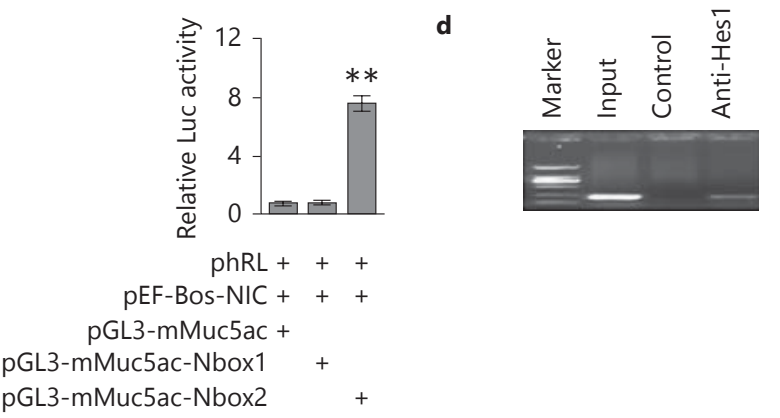

Hes1 could bind to the mouse Muc5ac promoter in mtCC1-2 cells using the ChIP assay using anti-Hes1. The co-precipitated chromatin DNA fragments were amplified by PCR using primers for the Muc5ac promoter region harboring the N-box $(-1,057$ to -857$)$. The results showed that the fragment with the Hes1-binding site was co-precipitated by the anti-Hes1 antibody (fig. 3d). The result further supported that the mouse Muc5ac promoter might be regulated by Notch signaling through Hes1dependent mechanisms. 
Fig.4. Effects of the Hes-binding sites on the human MUC5AC promoter. a Reporter assay. NCI-H292 cells were transfected with pGL3-hMUC5AC, and they were assayed as in figure 3a. b Scheme of the site-directed mutant of the human MUC5AC in which the N-boxes were disrupted. NCI-H292 cells were transfected independently with the wild type and mutant of the MUC5AC reporter constructs, and they were assayed as in figure 3a. c ChIP. Chromatin preparations were collected from NCI-H292 cells and were done as in figure $3 \mathrm{~d}$. Luc = Luciferase. a

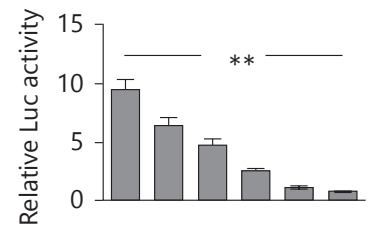

pGL3-mMUC5AC + + + + + phRL ++++++ pEF-Bos-NIC Hes1 ua - a

c

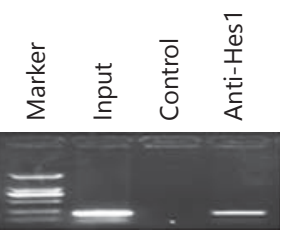

\section{b}
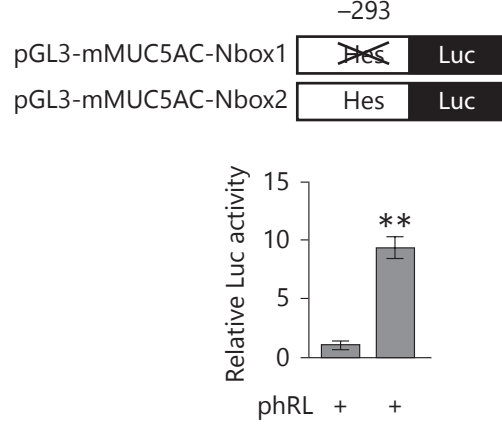

pEF-Bos-NIC + + PGL3-mMUC5AC + pGL3-mMUC5AC-Nbox2

\section{Effects of the Hes-Binding Sites on the Human} MUC5AC Promoter

We also amplified the human MUC5AC promoter $(-1,366 /+47)$ and constructed a reporter plasmid pGL3hMUC5AC. Gene report assays revealed that co-transfection of NCI-H292 cells with pEF-Bos-NIC gradually decreased human MUC5AC promoter activity (fig. 4a). Mutation of the N-box at position -293 increased the activity of the human MUC5AC promoter (fig. 4b). Similarly, ChIP assays showed that the human MUC5AC fragment with the Hes1-binding site ( -404 to -168$)$ could be coprecipitated by the anti-Hes1 antibody (fig. 4c). So, the human MUC5AC promoter might also be regulated by Notch signaling through Hes1-dependent mechanisms.

\section{Discussion}

In the present study, we described that Notch signaling regulated the expression of MUC5AC both in mouse mtCC1-2 cells and in human NCI-H292 cells. In vivo, a similar phenomenon is also observed in that MUC5AC expression increases in airway epithelial cells in a conditional inactivation of the essential Notch pathway component Pofut1 mouse model [9]. So, Notch signaling was implicated in the regulation of MUC5AC expression in airway secretary cells.

Then, we identified several Hes-binding site N-boxes in the $5^{\prime}$ region of both mouse and human MUC5AC promoters. This phenomenon suggested that these mo- tifs might have some regulatory role in the regulation of MUC5AC expression. Indeed, using a luciferase reporter assay, we showed that overexpression of NIC in both mtCC1-2 cells and NCI-H292 cells increased the transactivation of the MUC5AC promoter. Site-directed mutagenesis reporter assays revealed that Hes proteins might repress both mouse and human MUC5AC promoter activity. ChIP assays confirmed the binding of Hes 1 to the MUC5AC promoter regions. Thus, we believe that Notch signaling could regulate MUC5AC expression through an Hes1-dependent mechanism.

However, a recent study suggested that co-transfection with Hes5 full-length cDNA in LA4 cells also decreased the transcriptional activity of the MUC5AC promoter [9]. So, we speculate that the inhibitory effect of Notch on MUC5AC promoter activity might be shared by several bHLH factor downstream targets of Notch, including Hes1 and Hes5. The exact regulatory pattern may depend on the cell type, stimulatory factors, and the cellular surrounding environment, which remains to be further investigated. In addition, Notch signaling can regulate some posttranscriptional processes. Whether some posttranscriptional mechanisms are also involved in the regulatory process is still undetermined.

Kang et al. [12] showed that Notch signaling can induce EGFR and ERK phosphorylation and then upregulate Muc5ac expression. On the other hand, epidermal growth factor (EGF) stimulated generation of the Notch intracellular domain in an RBP-J-dependent manner 
[12]. Based on our results, we speculate that a bidirectional regulatory mechanisms may exist in the expression of MUC5AC through cross talk between Notch signaling and EGFR signaling.

The regulation of MUC5AC expression by Notch through Hes1-dependent mechanisms described here is likely to be relevant in the context of human disease. Failure of this mechanism may be related to airway epithelium malfunction that leads to mucus hypersecretion in conditions such as asthma. In asthmatic patients, mucus plugging of the airway lumen has been reported as one of the major contributing factors of fatal asthma $[13,14]$. Even in mild and moderate asthma, an increase in MUC5AC expression has been reported $[15,16]$. A recent study reported that stimulation of NCI-H292 cells with grass pollen allergen, which is a most common allergen that can lead to allergic rhinitis and asthma, results in the alteration of gene expression levels in 38 genes out of 74 genes that are involved in Notch signaling [17]. Meanwhile, expression of Notch1 and Hes1 mostly decreased, i.e. -2-fold and -3.3-fold respectively. However, no change was observed in the expression of Hes5 [17].
This is consistent with our findings that Hes 1 binds to the MUC5AC promoter regions. Notch signaling plays an important role in the pathogenesis of asthma by controlling $\mathrm{T}$ cell differentiation. However, only very limited data is available about whether Notch signaling can regulate the function of airway structural cells and then participate in the pathogenesis of asthma. Future experiments may further examine the effect of regulation of Notch signaling in airway epithelial cells on the phenotype of asthma in vivo. In conclusion, this study showed that Notch signaling can directly regulate MUC5AC promoter activity through Hes1-dependent mechanisms both in mouse mtCC1-2 cells and in human NCI-H292 cells, which may be identified as possible targets for pharmacotherapy of airway mucus hypersecretion in asthma.

\section{Acknowledgements}

The study was supported by grants from the National Natural Science Foundation of China (30900653).

\section{References}

$>1$ Lai HY, Rogers DF: Mucus hypersecretion in asthma: intracellular signaling pathways as targets for pharmacotherapy. Curr Opin Allergy Clin Immunol 2010;10:67-76.

$>2$ Voynow JA, Rubin BK: Mucins, mucus, and sputum. Chest 2009;135:505-512.

3 Fahy JV: Goblet cell and mucin gene abnormalities in asthma. Chest 2002;122:320S$326 \mathrm{~S}$.

4 Guseh JS, Bores SA, Stanger BZ, Zhou Q, Anderson WJ, Melton DA, Rajagopal J: Notch signaling promotes airway mucous metaplasia and inhibits alveolar development. Development 2009;136:1751-1759.

$>5$ Wong GT, Manfra D, Poulet FM, Zhang Q, Josien H, Bara T, Engstrom L, Pinzon-Ortiz M, Fine JS, Lee HJ, Zhang L, Higgins GA, Parker EM: Chronic treatment with the gamma-secretase inhibitor LY-411,575 inhibits beta-amyloid peptide production and alters lymphopoiesis and intestinal cell differentiation. J Biol Chem 2004;279:1287612882.

-6 Milano J, McKay J, Dagenais C, Foster-Brown L, Pognan F, Gadient R, Jacobs RT, Zacco A, Greenberg B, Ciaccio PJ: Modulation of notch processing by gamma-secretase inhibitors causes intestinal goblet cell metaplasia and induction of genes known to specify gut secretory lineage differentiation. Toxicol Sci 2004; 82:341-358.
7 Crosnier C, Vargesson N, Gschmeissner S, Ariza-McNaughton L, Morrison A, Lewis J: Delta-Notch signalling controls commitment to a secretory fate in the zebrafish intestine. Development 2005;132:1093-1104.

-8 van Es JH, van Gijn ME, Riccio O, van den Born M, Vooijs M, Begthel H, Cozijnsen M, Robine S, Winton DJ, Radtke F, Clevers H: Notch/gamma-secretase inhibition turns proliferative cells in intestinal crypts and adenomas into goblet cells. Nature 2005;435:959-963.

$\checkmark 9$ Tsao PN, Wei SC, Wu MF, Huang MT, Lin HY, Lee MC, Lin KM, Wang IJ, Kaartinen V, Yang LT, Cardoso WV: Notch signaling prevents mucous metaplasia in mouse conducting airways during postnatal development. Development 2011;138:3533-3543.

10 Qin H, Wang J, Liang Y, Taniguchi Y, Tanigaki K, Han H: RING1 inhibits transactivation of RBP-J by Notch through interaction with LIM protein KyoT2. Nucleic Acids Res 2004;32:1492-1501.

$>11$ Ou-Yang HF, Zhang HW, Wu CG, Zhang P, Zhang J, Li JC, Hou LH, He F, Ti XY, Song LQ, Zhang SZ, Feng L, Qi HW, Han H: Notch signaling regulates the FOXP3 promoter through RBP-J- and Hes1-dependent mechanisms. Mol Cell Biochem 2009;320:109-114.
12 Kang JH, Lee EH, Park SW, Chung IY: MUC5AC expression through bidirectional communication of Notch and epidermal growth factor receptor pathways. J Immunol 2011;187:222-229.

-13 Kuyper LM, Paré PD, Hogg JC, Lambert RK, Ionescu D, Woods R, Bai TR: Characterization of airway plugging in fatal asthma. Am J Med 2003;115:6-11.

14 Turner J, Jones CE: Regulation of mucin expression in respiratory diseases. Biochem Soc Trans 2009;37:877-881.

15 Ordoñez CL, Khashayar R, Wong HH, Ferrando $\mathrm{R}, \mathrm{Wu} \mathrm{R}$, Hyde DM, Hotchkiss JA, Zhang Y, Novikov A, Dolganov G, Fahy JV: Mild and moderate asthma is associated with airway goblet cell hyperplasia and abnormalities in mucin gene expression. Am J Respir Crit Care Med 2001;163:517-523.

16 Yasuo M, Fujimoto K, Tanabe T, Yaegashi H, Tsushima K, Takasuna K, Koike T, Yamaya M, Nikaido T: Relationship between calciumactivated chloride channel 1 and MUC5AC in goblet cell hyperplasia induced by interleukin-13 in human bronchial epithelial cells. Respiration 2006;73:347-359.

17 Röschmann KI, Luiten S, Jonker MJ, Breit TM, Fokkens WJ, Petersen A, van Drunen CM: Timothy grass pollen extract-induced gene expression and signalling pathways in airway epithelial cells. Clin Exp Allergy 2011;41:830-841. 